\title{
Polyribonucleotide 5'-Hydroxyl-Kinase Clp1
}

National Cancer Institute

\section{Source}

National Cancer Institute. Polyribonucleotide 5'-Hydroxyl-Kinase Clp1. NCI Thesaurus. Code C97530.

Polyribonucleotide 5'-hydroxyl-kinase Clp1 (425 aa, 48 kDa) is encoded by the human CLP1 gene. This protein plays a role in the modification of both mRNA and tRNA. 